\section{The Role of Community in Public Health}

The concept of "community" is central to the practice of public health. In developing and implementing policies and research studies, communities are often consulted, but key issues related to community involvement are not well defined. It is difficult sometimes even to define a community. Communities are characterized by common elements such as place of birth, culture, religion, geography and sexual orientation. Nevertheless, within communities, there is considerable diversity for example as to gender, education, socio-economic status and immigration history.

The manner in which the community should participate in health policy and research is not clear. First, there is the issue of representativeness. Persons or groups may express personal opinions that are not in fact shared by many, or even most, other members of the community. A second, and somewhat related issue, is accountability. In general, community representatives are not elected or appointed by a wider body and, therefore, the manner in which they are accountable to the larger community is usually not defined. There are mechanisms by which this can be accomplished. An example is the community advisory board where community members can provide input to research projects.

It is important that an individual representing community perspectives possess professional or personal credentials that lend weight to the opinions expressed. There is a tendency to reflexively confer legitimacy on a person or group who purports to represent a community. In this way, an individual expressing an opinion that is problematic, ill-informed or even retrogressive may be given credence because of a claim to representativeness. The credibility conferred can result in a tyranny of political correctness.

In the field of HIV in particular, community involvement has been both useful and problematic. Community-based advocacy has been a powerful driver of policy decisions related to research, clinical management and prevention policy. Nevertheless, some of this input has not been helpful. The preventive interventions developed by the US Centers for Disease Control referred to as DEBIs (Diffusion of Effective Behavioral Interventions) have faced considerable community resistance despite their demonstrated impact in independent studies and evaluations. Beliefs about effective prevention approaches based on personal experience or anecdote are important to consider but need to be interpreted in the context of scientific evidence. Given the enormous challenge presented by primary and secondary prevention of HIV, good ideas, even if widely held, are simply not good enough.

Involving communities is critical to the success of public health efforts. However, we must think carefully about how to achieve meaningful and effective involvement.

Robert S. Remis
Interim Scientific Editor

\section{Le rôle de la communauté en santé publique}

La notion de "communauté » est au cour de la pratique en santé publique. On consulte souvent les communautés lorsqu'on élabore et que l'on met en œuvre des politiques et des études de recherche, mais les grands enjeux de l'engagement communautaire ne sont pas bien définis. Il est même parfois difficile de définir ce qu'est une communauté. Les communautés se caractérisent par des éléments communs, comme le lieu de naissance, la culture, la religion, la géographie et l'orientation sexuelle. On trouve néanmoins au sein des communautés une diversité considérable, par exemple selon le sexe, l'instruction, le statut socioéconomique et les antécédents d'immigration.

La manière dont la communauté devrait participer aux politiques et à la recherche en santé n'est pas claire. Il y a d'abord la question de la représentativité. Des individus ou des groupes peuvent exprimer des opinions personnelles qui, dans les faits, ne sont pas partagées par une grande partie - voire la majeure partie - de leur communauté. Une deuxième question, qui va un peu dans le même sens, est celle de la responsabilisation. En général, les représentants communautaires ne sont ni élus, ni désignés par un organisme plus large; en conséquence, la manière dont ils rendent des comptes à la communauté n'est habituellement pas définie. Il existe des mécanismes pour ce faire : le conseil consultatif communautaire, où les membres d'une communauté peuvent se prononcer sur des projets de recherche, en est un exemple.

Il est important qu'une personne qui représente les perspectives de sa communauté possède des qualifications professionnelles ou personnelles qui donnent du poids aux opinions qu'elle exprime. On a tendance à conférer automatiquement une légitimité à une personne ou un groupe censé représenter une communauté. Ainsi, une personne qui exprime une opinion douteuse, mal informée ou même régressive peut se voir accorder une crédibilité parce qu'elle prétend à la représentativité. La crédibilité ainsi accordée peut mener à la tyrannie de la rectitude politique.

Dans le domaine du VIH en particulier, l'engagement communautaire est à la fois utile et problématique. Le plaidoyer communautaire est un puissant moteur dans les décisions stratégiques liées à la recherche, à la prise en charge clinique et aux politiques de prévention. Néanmoins, certaines de ces prises de parole n'aident en rien. Le projet d'interventions préventives des Centers for Disease Control américains connu sous l'acronyme DEBI (pour « diffusion des interventions efficaces visant à modifier les comportements ») fait face à une résistance communautaire considérable malgré son impact, prouvé par des études et des évaluations indépendantes. Lorsqu'il s'agit de l'efficacité des approches de prévention, il est important d'examiner les croyances fondées sur l'expérience personnelle ou anecdotique, mais il faut interpréter ces croyances à la lumière des preuves scientifiques. La prévention primaire et secondaire du VIH pose un défi énorme; les bonnes idées, même si elles sont largement répandues, ne sont simplement pas suffisantes.

L'engagement des communautés est indispensable au succès des efforts de santé publique. Mais nous devons réfléchir soigneusement à la manière d'obtenir un engagement communautaire sérieux et efficace.

Robert S. Remis

Rédacteur scientifique intérimaire 